\title{
Itinerario+
}

\section{un modelo socioeducativo integral para el alumnado de Formación Profesional Básica}

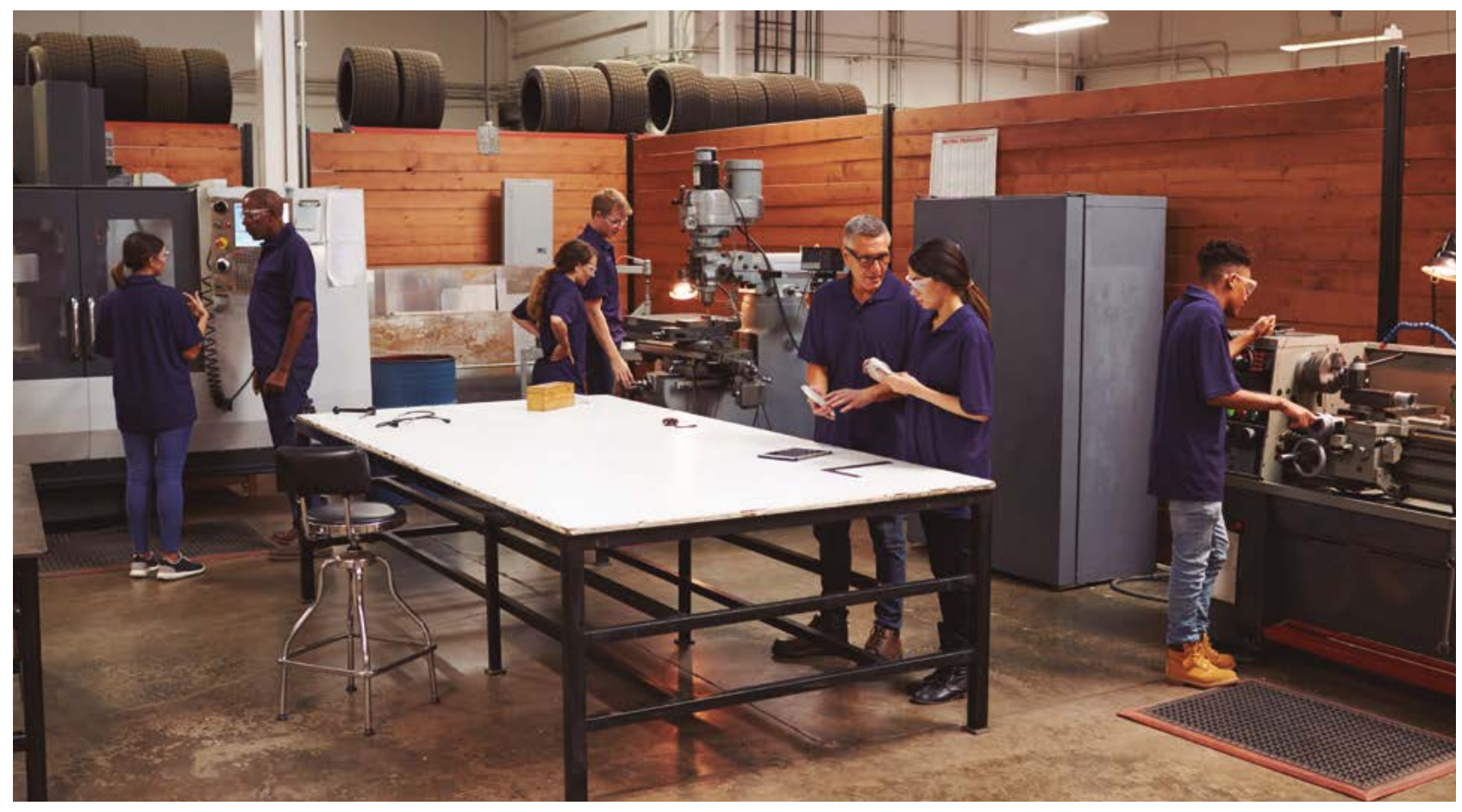

Itinerario + es un proyecto que busca demostrar que trabajando de forma integrada los aspectos formales y no formales, personales y profesionales, así como sociales y comunitarios se puede cambiar la trayectoria vital de jóvenes procedentes de contextos desfavorecidos, generando un modelo innovador que asegura que los y las jóvenes que pasan por él son más competentes desde un punto de vista

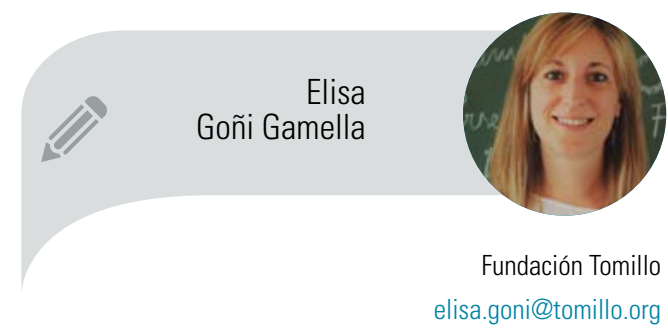
socioemocional, son más empleables y cuentan con un plan de vida que les permite tomar decisiones informadas sobre su futuro. 


\section{El alumnado de Formación Profesional Básica}

En cualquier centro de Educación Secundaria podemos encontrar estudiantes con dificultades: de aprendizaje, de relación, económicas, de comportamiento... Si hablamos de un centro de Formación Profesional (FP), como el de la Fundación Tomillo, en el que dos tercios del alumnado es de Formación Profesional Básica (FPB), son muchos los alumnos y las alumnas con algún tipo de dificultad.

Con este escenario, nos encontramos estudiantes con quienes ya sabemos que algunas de las metodologías más tradicionales no funcionan. No funcionan las clases magistrales, no funcionan los deberes en casa, no funciona que todo el grupo lleve el mismo ritmo, no funciona la memorización para volcarla en un examen... Hablamos de jóvenes que llegan muy dañados del sistema educativo tradicional, con experiencias académicas agridulces y una creencia arraigada de que no valen, de que no pueden, de que son estudiantes -y personas - de segunda.

Este punto de partida hace que nuestra labor docente en Tomillo sea distinta. Es muy importante cómo nos aproximamos al alumnado cuando llega, cómo vamos desmontando creencias previas, curando heridas para que puedan reconstruirse como estudiantes y como personas. Durante años, hemos ido incluyendo metodologías y formas de acompañar dentro y fuera del aula que han funcionado y, después de analizar el impacto de estas prácticas, ordenarlas y darles forma tenemos, finalmente, Itinerario+.

Pero, ¿por qué es diferente Itinerario +?

\section{Atención personalizada}

El primer contacto que tiene un/a joven que empieza FPB en Tomillo es la tutoría inicial; una entrevista personal a la que, posteriormente, se une su familia. En ella conoce a su tutor/a, el enfoque y objetivos del curso y la fundación. Muchos chicos y chicas vienen a la entrevista con ilusión; quieren cambiar. Otros vienen con escepticismo, pensando que la FPB va a ser lo mismo. Empiezan la en-

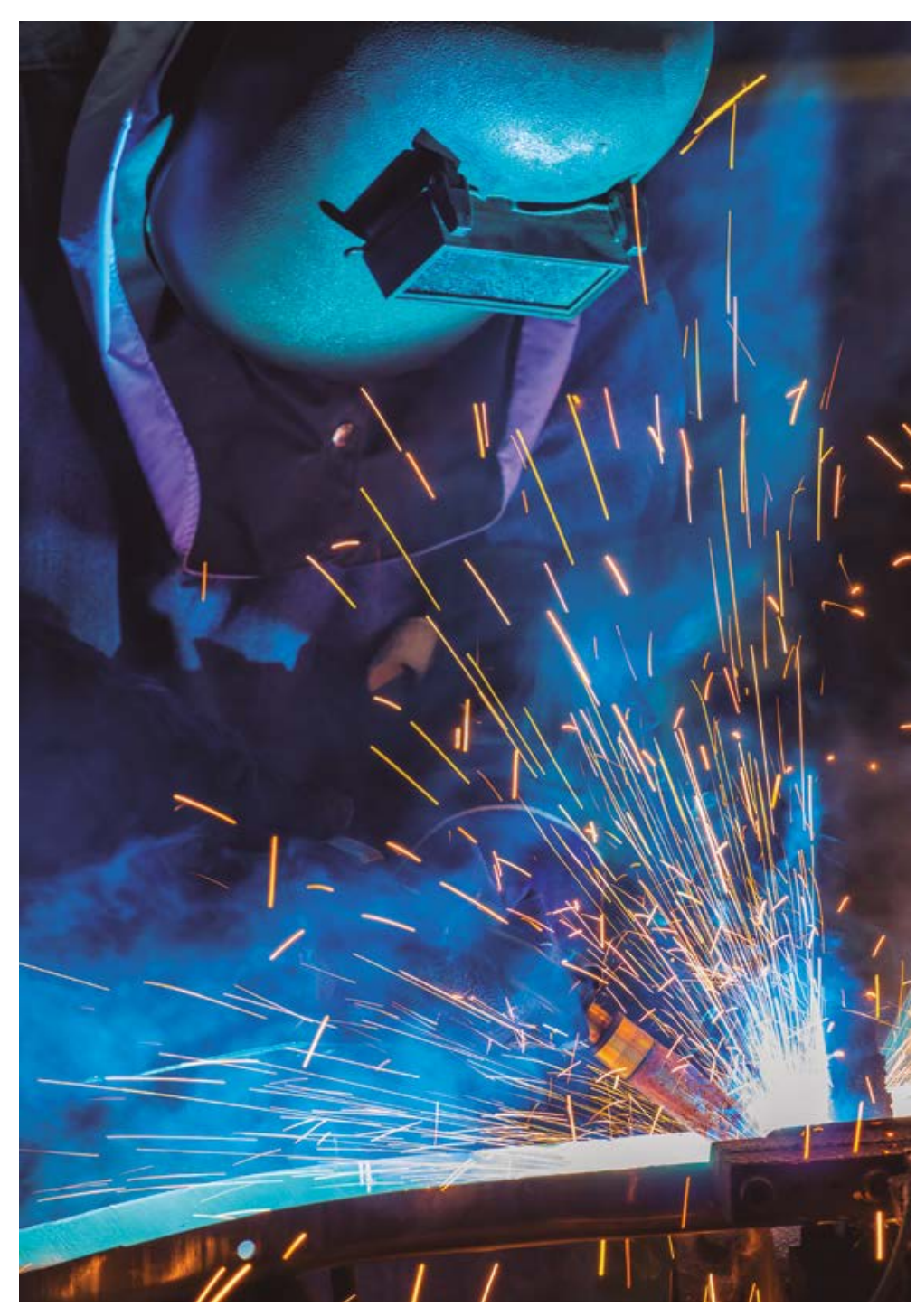

trevista muy serios, pero poco a poco se van relajando y se sorprenden cuando les decimos que no nos importa lo que haya pasado anteriormente, que es el momento de dejar atrás el pasado académico, que a partir de ahora todo puede ser diferente y que en Tomillo no nos importa lo que hayan hecho antes, sino lo que escojan y decidan hacer a partir de ahora. Y nosotros estamos aquí para acompañarlos en el proceso. Así, se empiezan a crear lazos de confianza.

\section{Desarrollo de competencias socioemocionales}

Cuando entran a clase por primera vez, ven un espacio que se parece más a un espacio de coworking que a un aula, con distintas zonas de trabajo y, lo más sorprendente, compartiendo aula con otro grupo de un perfil profesional diferente al suyo. 


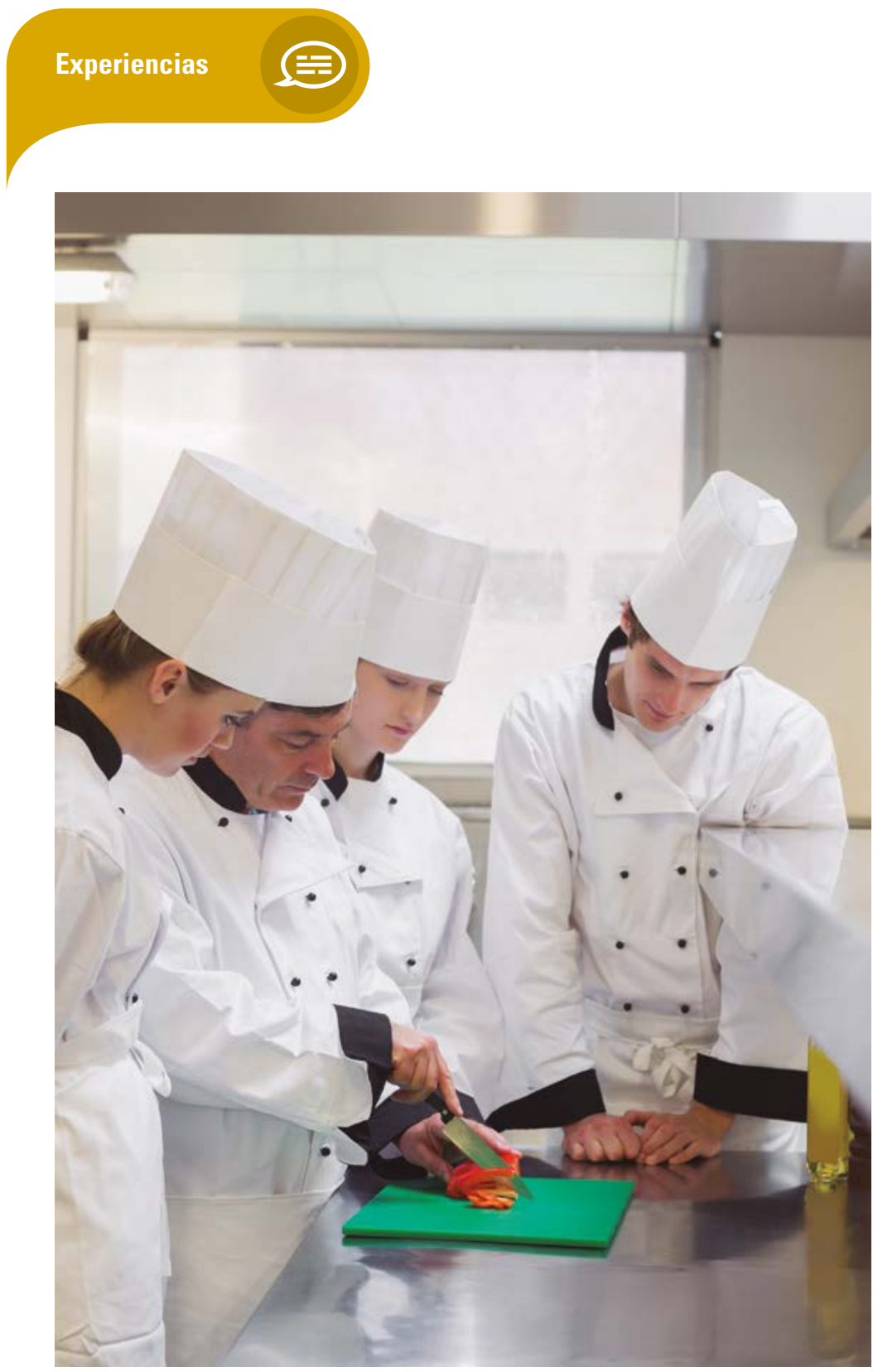

principio esto les crea cierta ansiedad; no están acostumbrados a tomar este tipo de decisiones, normalmente ya se lo dan hecho. También les es complicado priorizar tareas e incluir las que no les gustan, pero es una rutina que va mejorando diariamente tanto por la experiencia que van adquiriendo como por el feedback continuo del profesorado.

El primer proyecto que trabajamos es la Acogida. Es la parte más importante del curso porque fortalece el vínculo con sus profesores/as y compañeros/as, y sienta las bases emocionales de partida del alumnado para el curso. Esto es, cuando un/a estudiante comienza con miedo a fracasar de nuevo, en actitud defensiva, es casi imposible que pueda avanzar. Al trabajar las competencias socioemocionales y relacionales con dinámicas, ganan confianza en sí mismos/as y en los demás, y empiezan a abandonar el papel que desempeñaban en su antigua clase.

Además, durante la acogida trabajamos las herramientas digitales que vamos a utilizar durante el curso: correo electrónico, Classroom, Drive... Así, cuando comenzamos a trabajar la parte técnica, se sienten más seguros y ponen el foco en las habilidades profesionales.

Empiezan la mañana con una actividad que trabaja las competencias personales. Les cuesta mucho, porque hay que mirarse por dentro; no están acostumbrados y en ocasiones les asusta lo que puedan encontrar. Otras veces realizamos dinámicas para relacionarse con sus pares de otra forma que no sea la académica y establecen lazos que les servirán para trabajar colaborativamente y crear un clima de confianza en el aula.

Luego organizamos el día con la Agenda Consciente. Esta parte es muy importante, pues el alumnado tiene bastante libertad a la hora de organizar y escoger sus tareas. Para hacer la agenda necesita tener en cuenta qué docentes van a estar en el aula, si hay píldoras formativas, si hay algún taller de orientación vocacional, los productos que tiene que presentar y organizarse con los compañeros y compañeras si el trabajo es en equipo. Al

\section{Proyectos integrales}

A lo largo del curso, los y las estudiantes trabajan en proyectos integrales que les permiten adquirir y desarrollar conocimientos, habilidades y competencias de manera global, con sentido y propósito. Inicialmente son proyectos más guiados y a medida que su autonomía crece, va aumentando la capacidad de elección sobre qué aprenden, cómo y cuándo.

Además, los proyectos implican aprender fuera del aula frecuentemente: salimos a explorar el barrio, entrevistar a personas del entorno, visitar empresas, viajar a otras ciudades cercanas. Por un lado, aprender fuera de las cuatro paredes del aula les permite hacerlo de manera experiencial; se sienten más activos, más motivados. Por otro lado, visitar empresas y entrar en contacto con personas de realidades muy diferentes a la suya 


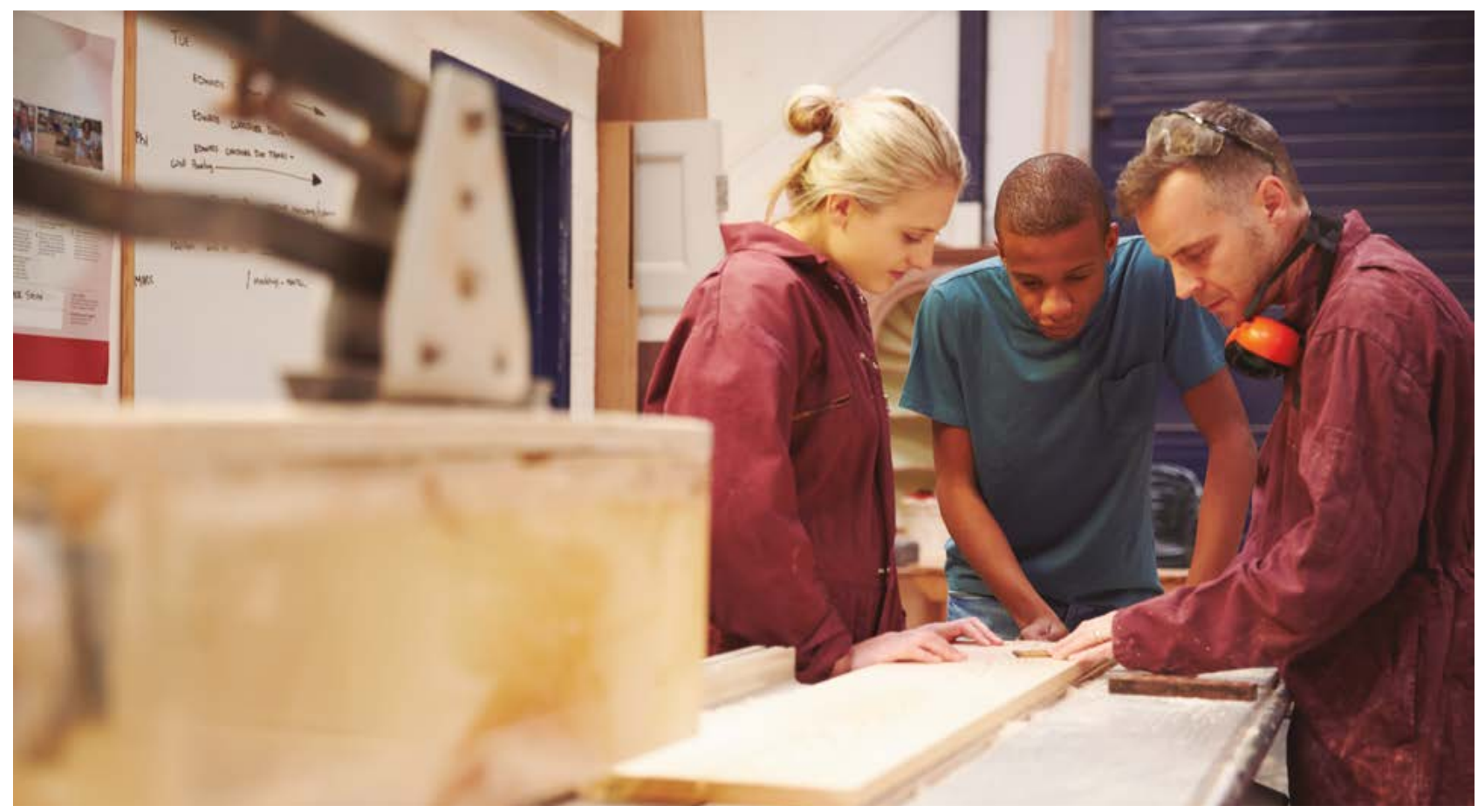

les permite ampliar sus perspectivas, conocer otros caminos que antes no sabían que existían. Algunos/as se sienten abrumados/as porque ven muy lejana esa realidad; es normal, es parte del proceso. Otros/as sienten inspiración y curiosidad; descubren que hay más posibilidades de las que conocían hasta ahora, y empiezan a plantearse nuevas aspiraciones.

Por otro lado, durante los proyectos, las familias reciben Ilamadas del profesorado, no para decirles que su hijo/a ha sido expulsado/a, sino para compartir con ellos una fortaleza de su hijo/a, un avance significativo, un cambio de actitud positivo... Las etiquetas previas se van difuminando, van desapareciendo a ojos del alumno y de su familia. Poco a poco se van desmontando las creencias y empieza a cobrar nitidez un nuevo potencial.

\section{Desdibujando la línea entre la educación formal y no formal}

Los proyectos se complementan con experiencias de aprendizaje no formal que enriquecen su desarrollo integral y activan su motivación. Así, ya sea por la tarde después de clase o durante el horario escolar, los alumnos tienen la posibilidad de realizar píldoras, talleres o trayectos formativos relacionados con el arte, el deporte, la naturaleza, la tecnología o la música. Estos espacios sirven para que los alumnos exploren desde un escena-

\author{
Se van relajando y se sorprenden cuando les \\ decimos que en Tomillo no nos importa lo que \\ hayan hecho antes, sino lo que escojan y \\ decidan hacer a partir de ahora. Y nosolros/as \\ estamos aquí para acompañarlos en el proceso
}

rio de aprendizaje diferente y desarrollen competencias personales. Pero, además, estos espacios abiertos por la tarde suponen para muchos un lugar seguro, donde se sienten en confianza, acompañados y escuchados. Hay estudiantes que no quieren volver a casa para no enfrentarse a la realidad de su hogar, y se sienten agradecidos/as de tener a su disposición este recurso disponible para ellos/as.

\section{Evaluación para el aprendizaje}

Otro de los grandes cambios que experimenta un/a joven cuando entra en Tomillo es la forma de evaluación. Viene acostumbrado a ser una nota, un número, una calificación que lo ha etiquetado a lo largo de su vida académica. Cuando llega a Tomillo, se descoloca al ver que no hay notas, no hay boletines que etiqueten al estudiante en las diferentes asignaturas, sino informes competenciales. De pron- 


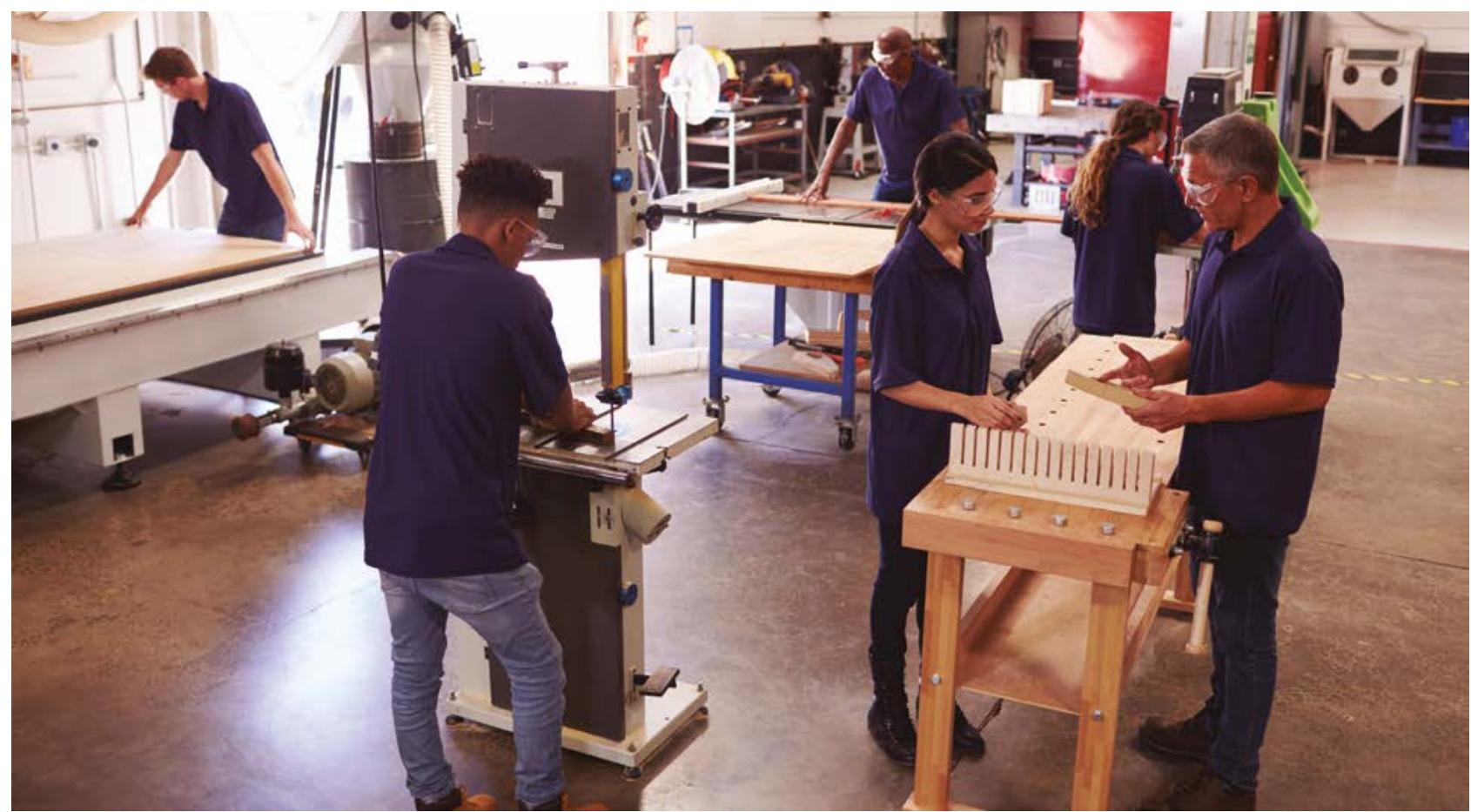

Hay un orientador presente en el aula que

vela por que su experiencia formativa sea

personalizada, quien orienta y guía al alumno

en su toma de decisiones y en la exploración de

sus vocaciones to, descubre que tiene un papel activo en su propia evaluación: aprende a usar un porfolio donde recoge evidencias de su aprendizaje y de su desarrollo competencial a lo largo de todo el proyecto; utiliza un diario de aprendizaje en el que reflexiona sobre su progreso, sus avances y las estrategias que le ayudan a aprender más y mejor; recibe feedback diario de sus profesores que le permite ser consciente de sus fortalezas, sus progresos y sus áreas de mejora. El ciclo de evaluación carece de exámenes; es el/la estudiante quien selecciona las evidencias que le permiten identificar en qué grado ha alcanzado cada objetivo de aprendizaje curricular y socioemocional y las presenta a sus compañeros/as, que le ofrecen feedback posteriormente. El equipo docente también recoge evidencias de progreso y logro que completa con las evidencias del alumnado. El ciclo de evaluación culmina con una reflexión guiada donde el/la joven compara su autoevaluación con la evaluación recibida por sus compañeros/as y profesores/as en los objetivos de aprendizaje y competencias socioemocionales en base a una rúbrica de competencias. Tras este análisis, el alumnado se marca dos objetivos a trabajar en el siguiente proyecto y diseña un plan de acción. Este marco de evaluación contribuye a que estos chicos y chicas, que llegaron pensando que no valían y que no podían, desarrollen su propia brújula interna y se empoderen de su proceso de aprendizaje. Son mucho más conscientes de qué aprenden, cómo lo aprenden y en qué necesitan centrar sus esfuerzos.

\section{Prácticas profesionales en empresas}

Todas estas acciones cobran sentido cuando el/la alumno/a va a prácticas a una empresa. Es ahí cuando despliega todo su potencial. El alumno/a, que durante el primer trimestre llegaba siempre tarde, no se quitaba la capucha en clase y se ocultaba tras la pantalla del ordenador, ahora demuestra (se demuestra) que es capaz de acudir puntual a trabajar, capaz de aplicar lo aprendido en el taller del aula en una empresa real, capaz de perfeccionar sus técnicas profesionales y aprender de y con otros. No sería posible que los y las alumnas tuvieran una ex- 


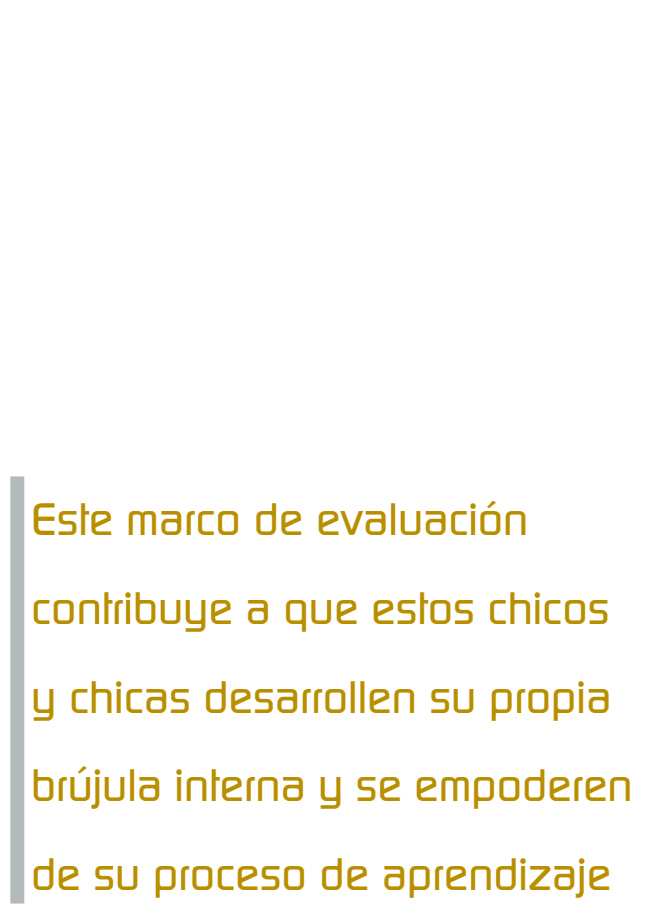

periencia de aprendizaje tan significativa y tan aprovechada en la empresa si previamente no hubiéramos acompañado su desarrollo socioemocional y técnico a lo largo del curso.

\section{Orientación vocacional}

Por último, a lo largo del trayecto formativo de un/a alumno/a en Tomillo, hay un/a orientador/a presente en el aula que vela por que su experiencia formativa sea personalizada, quien orienta y guía al alumno/a en su toma de decisiones y en la exploración de sus vocaciones. De esta manera, el alumno/a, que inicialmente entró en FPB con una mochila cargada de experiencias de fracaso, sale siendo consciente de cuál es su siguiente paso; siendo consciente de sus logros, sus fortalezas y aceptando sus limitaciones; sale siendo capaz de tomar decisiones personales y profesionales conscientes e informadas; en definitiva, sale de Tomillo con un plan de vida.

\section{HEMOS HABLADO DE}

\section{Educación integral; evaluación para el aprendizaje; orientación vocacional; Formación Profesional Básica; aprendizaje socioemocional; aprendizaje basado en proyectos.}

Este artículo fue solicitado por PADRES Y MAESTROS en septiembre de 2020, revisado y aceptado en febrero de 2021.

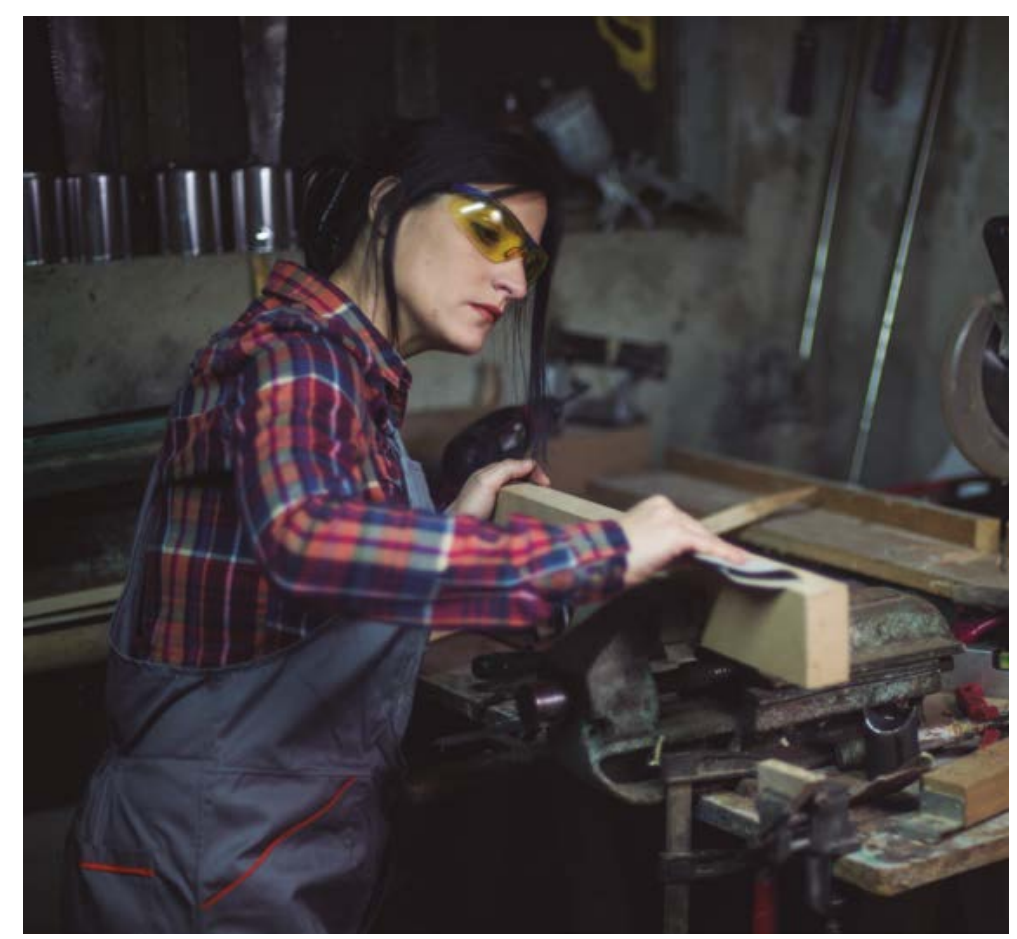

Una apuesta por el desarrollo integral de la persona

De esta manera, podríamos concluir que Itinerario+ es la suma de esfuerzos, metodologías, palancas y recursos planificados y secuenciados estratégicamente con el objetivo de demostrar que trabajando de forma integrada los aspectos formales y no formales, personales y profesionales, así como sociales y comunitarios, se puede cambiar la trayectoria vital de jóvenes procedentes de contextos desfavorecidos, generando un modelo innovador que asegura que los jóvenes que pasan por él son más competentes desde un punto de vista socioemocional, son más empleables y cuentan con un plan de vida que les permite tomar decisiones informadas sobre su futuro •

\section{DD DARR SABER MÂS}

Brookfield, S. D. (1995). Becoming a Critically Reflective Teacher. San Francisco, CA: Jossey-Bass.

CAsel. (s. f.). Marco competencial CASEL. Recuperado de https://casel.org/

CAS. (s. f.). The UDL guidelines. Recuperado de http://udlguidelines.cast.org/

Hattie, J. (2008). Visible Learning. Abingdon, Oxon: Routledge. 